\title{
An Unusual Cause of Dementia: Essential Diagnostic Elements of Corticobasal Degeneration-A Case Report and Review of the Literature
}

\author{
F. Mastrolilli, A. Benvenga, L. Di Biase, F. Giambattistelli, L. Trotta, G. Salomone, \\ L. Quintiliani, D. Landi, J. M. Melgari, and F. Vernieri
}

Department of Neurology, “Campus Biomedico” University, Via Álvaro del Portillo, 21-00128, Rome, Italy

Correspondence should be addressed to F. Mastrolilli, f.mastrolilli@unicampus.it

Received 15 January 2011; Revised 21 April 2011; Accepted 18 May 2011

Academic Editor: Florinda Ferreri

Copyright ( 2011 F. Mastrolilli et al. This is an open access article distributed under the Creative Commons Attribution License, which permits unrestricted use, distribution, and reproduction in any medium, provided the original work is properly cited.

\begin{abstract}
Corticobasal degeneration (CBD) is an uncommon, sporadic, neurodegenerative disorder of mid- to late-adult life. We describe a further example of the pathologic heterogeneity of this condition. A 71-year-old woman initially presented dysarthria, clumsiness, progressive asymmetric bradykinesia, and rigidity in left arm. Rigidity gradually involved ipsilateral leg; postural instability with falls, blepharospasm, and dysphagia subsequently developed. She has been previously diagnosed as unresponsive Parkinson's Disease. At our clinical examination, she presented left upper-arm-fixed-dystonia, spasticity in left lower limb and pyramidal signs (Babinski and Hoffmann). Brain MRI showed asymmetric cortical atrophy in the right frontotemporal cortex. Neuropsychological examination showed an impairment in visuospatial functioning, frontal-executive dysfunction, and hemineglect. This case demonstrates that association of asymmetrical focal cortical and subcortical features remains the clinical hallmark of this condition. There are no absolute markers for the clinical diagnosis that is complicated by the variability of presentation involving also cognitive symptoms that are reviewed in the paper. Despite the difficulty of diagnosing CBD, somatosensory evoked potentials, motor evoked potentials, long latency reflexes, and correlations between results on electroencephalography (EEG) and electromyography (EMG) provide further support for a CBD diagnosis. These techniques are also used to identify neurophysiological correlates of the neurological signs of the disease.
\end{abstract}

\section{Introduction}

Corticobasal degeneration (CBD) is an uncommon, sporadic, neurodegenerative disease described for the first time by Rebeiz et al. [1]. It can be associated with an extraordinary variety of motor, sensory, behavioural, and cognitive symptoms [2].

It is an asymmetrical parkinsonism affecting a limb, typically an arm: rigidity is the most common manifestation of the parkinsonian syndrome followed by bradykinesia, gait disorder (postural instability and falls), and tremor; asymmetrical limb dystonia is common as well. Other cardinal signs include higher cortical dysfunctions such as apraxia (limb more common than orofacial, eyelid-opening). Dementia, progressive nonfluent aphasia, speech apraxia, progressive-supranuclear-palsy- (PSP-) like syndrome and posterior cortical atrophy syndrome are other presentations of CBD $[3,4]$.

CBD is a tauopathy (characterised by abnormal deposition of the microtubule-associated protein tau), similar to frontotemporal dementia and progressive supranuclear palsy (PSP) [5].

The typical pathological findings in CBD include focal asymmetric cortical atrophy, nigral degeneration, taupositive neuronal, and glial lesions in both gray and white matters [6].

To achieve more accurate clinical diagnosis, neuropsychological, electrophysiological, and imaging methods could be applied to differentiate this disease from the other parkinsonism syndromes $[3,4]$.

In comparison with other neurological diseases, the symptoms of CBD are particularly difficult to understand and the patients have considerable difficulties in describing 
their experience. A better understanding of the disease may help clinicians to make diagnosis, providing patients with comprehensive information about prognosis and difficulties they will encounter during the course of the disease, improving their quality of life as well as their careers.

\section{Case Report}

A 71-year-old woman, primary school graduate, formerly farmhand, was referred to our Department of Neurology with left rigid-akinetic syndrome and cognitive dysfunction.

She had no previous medical or family neurological history; she just reported in the previous months frequent falls and postural instability.

She initially presented with slowly progressive dysarthria and speech abnormalities two years before. Cognitive symptoms included impairment of spoken-language production and attention/concentration deficits.

Several months later clumsiness and rigidity in her left upper limb appeared. Her main complaint was difficulty in using her left arm and hand, which gradually progressed. The limb became severely rigid and adopted a dystonic posture associated with pain and functional disability.

Her clinical features slowly deteriorated and, 1 year later, involved also the left lower limb with gait disorder associated to postural instability and falls. Subsequently, the patient underwent brain MRI and 18F-fluorodeoxyglucose-positron emission tomography (PET).

Brain MRI showed moderate atrophy, more pronounced on the right side. She has been previously diagnosed as Parkinson's Disease but with no response to levodopa or dopaminergic medications.

Asymmetrical hypometabolism involving the right frontal cortex was also confirmed by PET.

Her clinical symptoms gradually progressed and, four months later, blepharospasm and mild dysphagia appeared.

When she was admitted to our Neurological Centre, the neurological examination revealed: blepharospasm; hypomimia and asmmetric bradykinesia; left upper-armfixed-dystonia; spasticity in left lower limb and pyramidal signs (Babinski, Hoffmann, and grasp reflex); moderate disturbance of gait with short steps, tendency to drag her left leg, bradykinesia, and propulsion requiring assistance.

We performed a complete neuropsychological examination (for more details see Table 1). Mini Mental State Examination score was 23/30. The other tests revealed impairment in visuospatial abilities, severe visuospatial neglect (Figure 1), constructional and ideomotor apraxia, poor word fluency with mild visual confrontation anomia, and nonverbal oral apraxia. Learning and memory were minimally affected.

Brain MRI showed asymmetric cortical atrophy in the right frontotemporal cortex (Figure 2).

No abnormalities were found on EEG examination, even if, a deeper analysis showed a focal slow wave activity in the right parietotemporal area.

Related to clinical features, based upon diagnostic criteria proposed by Boeve et al. [4], she has been diagnosed as "corticobasal degeneration (CBD)".


Figure 1: Neuropsychological examination show emineglect (Raven's colored matrices and barrage test) and constructional apraxia (Rey-Osterrieth complex figure).
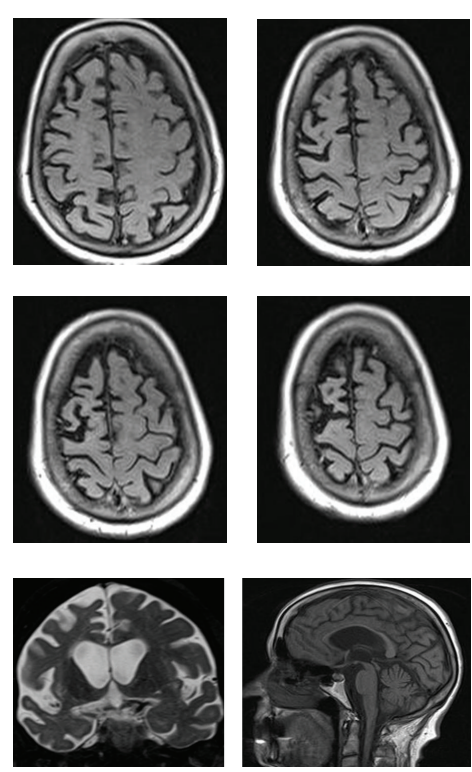

FIGURE 2: Axial, T2-weighted and fluid-attenuated inversion recovery (FLAIR), MRI images of the brain, demonstrating asymmetric cortical atrophy in the right hemisphere of the patient.

The diagnosis was based on the gradual onset of a parkinsonian disorder associated with cortical dysfunctions and other supportive features such as cognitive dysfunction, asymmetric atrophy on MRI imaging, and asymmetric hypoperfusion on PET.

\section{Discussion and Review of the Literature}

3.1. Clinical Presentation. Clinically CBD begins in the sixth, seventh, or eighth decade [7], with slight predilection for women $[8,9]$. Typically the primary symptoms develop in a profoundly asymmetric way, affecting either one arm or, less frequently, a leg, which appears to be rigid, dystonic, akinetic, or apraxic. Clinical features include a series of motor, cognitive and neuropsychiatric symptoms, that can 
TABLE 1: Complete neuropsychological examination performed by the patient.

\begin{tabular}{|c|c|c|c|}
\hline & Obtained score & Cut-off & Result \\
\hline \multicolumn{4}{|l|}{ Rey Auditory Learning Test } \\
\hline Immediate recall & 40 & 28,53 & Normal \\
\hline Delayed recall & 8 & 4,69 & Normal \\
\hline Recognition recall & 14/15, 4/30; accuracy \% 95 & 92 & Normal \\
\hline Digits forward & 5 & $7 \pm 2$ & Below normal \\
\hline Digit backward & 2 & $5 \pm 2$ & Below normal \\
\hline Corsi span Forward & 3 & $7 \pm 2$ & Below normal \\
\hline Corsi span Backward & 2 & $5 \pm 2$ & Below normal \\
\hline $\begin{array}{l}\text { Rey-Osterrieth Complex figure } \\
\text { delayed recall }\end{array}$ & 2,5 ; correct 9,5 & 9,46 & Normal \\
\hline \multirow[t]{2}{*}{ Barrage Test } & $D x(26 / 30) ; S x(5 / 30)$ & 59 & Below normal \\
\hline & Time 95" & $>105^{\prime \prime}$ & \\
\hline \multirow[t]{2}{*}{ Deux Barrage } & $5 / 13,22 / 67 ;$ accuracy $\% 57$ & 95 & Below normal \\
\hline & Time $210^{\prime \prime}$ & $>133^{\prime \prime}$ & $\begin{array}{l}\text { Prolonged time of } \\
\text { execution }\end{array}$ \\
\hline Rey-Osterrieth Complex figure Test & 4,5 ; correct 7 & 28,87 & Below normal \\
\hline Ideomotor Praxia & 8,5 & 9 & Below normal \\
\hline Buccofacial apraxia & 10 & 9 & Normal \\
\hline Raven's colored Matrices & 6 ; correct 10,5 & 18,96 & Below normal \\
\hline \multicolumn{4}{|l|}{ Verbal Fluency } \\
\hline Phonetc cues & 10 ; correct 18,6 & 17,35 & Normal \\
\hline Semantic cues & 12 ; correct 14,3 & 10,3 & Normal \\
\hline Naming (B.A.D.A.) & 24 & 28 & Below normal \\
\hline
\end{tabular}

be explained by impairment of the cortical and subcortical structures.

Motor symptoms include progressive asymmetric rigidakinetic parkinsonism usually involving the upper limbs, without resting tremor [10], focal stimulus-sensitive or action myoclonus $[4,11]$, blepharospasm, speech abnormalities, gait disorder with postural instability and falls, and asymmetric limb dystonia, generally of the upper limbs, sometimes evolving towards the development of a dystonic clenched fist $[3,12]$.

Eye movements are usually preserved, although a delay in the initiation of saccades may occur, in absence of pursuit and optokinetic nystagmus impairment $[13,14]$.

Involvement of higher cortical functions results in often symmetric ideomotor apraxia, firstly affecting the limb, then, as the disease progresses, eyelid-opening, tongue, lips. The alien-limb phenomenon, that is seen in $50 \%$ of the cases [15], can be defined as "a circumstance in which one of the patient's hands behaves in a way which the patient finds foreign, alien or at least uncooperative"; it commonly cooccurs with cortical sensory loss $[11,16]$.

Cognitive decline is a common feature of the disorder [17], occasionally the presenting feature of the disease [18]. The prominent characteristics are impairment of spokenlanguage production (typically nonfluent aphasia), frontal executive impairment, calculation and visuospatial skills impairment, whereas semantic and episodic memory may be spared [17].

Neuropsychiatric symptoms may include depression, apathy, anxiety, irritability, disinhibition, delusions, and obsessive compulsive disorder [19].

3.2. Electrophysiology. Abnormalities on magnetoencephalography [20], an exaggerated electromyographic-electromyographic (EMG-EMG) coherence [21], and an alteration in cortical excitability evaluated by means of transcranial magnetic stimulation (TMS) [22] were noted in patients with CBD [23]. On the other hand, conventional electroencephalography (EEG) may be normal when the first clinical symptoms appear, and often remains unchanged as the disease progresses. Nevertheless, an unilateral slowing may be evident in some patients, which may occasionally generalise to the whole cortex as the disease evolves [24, 25]. In a study involving six patients, Vion-Dury and coworkers, using a quantitative standard EEG (EEGq) with spectral analysis, found indeed the occurrence of several EEG abnormalities (generally enhanced by hyperventilation or intermittent photic stimulation), such as an increase of slow rhythms (delta or theta frequency range) and occasionally the occurrence of sharp waves [25]. These abnormalities were 
lateralised in five patients (more often after hyperventilation) and were bilateral in one, confirming the asymmetrical features of CBD [25]. Moreover, Huang et al. showed that the EEG recordings with jerk-locked back average do not present any jerk-locked cortical potentials [23].

In CBD, the cortical sensory evoked potentials (SEPs) are not enlarged as in cortical reflex myoclonus, and backaveraged cortical potentials do not precede each myoclonic jerk [26-28]. Clinical and imaging evidence suggests that the localized parietal cortical damage is a pivotal factor for the absence of a giant SEP in these patients [29]. An asymmetric alternation of inhibitory and excitatory balance at the level of cortical neurons leading to a particularly enhanced cortical excitability may moreover play an important role in the generation of myoclonus $[27,28]$. The loss of the inhibitory input from the somatosensory cortex to the relatively intact motor cortex, which results from the prominent asymmetric parietal atrophy, may give rise to the asymmetric hyperexcitable motor cortex without giant SEP [30, 31], even-though the existence of an alternative hyper-excitable thalamo-cortical pathway cannot be excluded [32]. In effect, motor cortex disinhibition has been clearly demonstrated in CBD by means of TMS applied in several paradigms in different neurophysiological studies [32-34]. By applying single pulse-TMS, Lu and coworkers discovered a relatively higher motor evoked potential (MEP) amplitude and a significantly shorter cortical silent period in the affected hand of CBD patients [26]. They therefore supposed that the relatively enlarged MEP may be explained postulating that an increased number of motorneurons are being recruited by the descending volleys from the motor cortex [26], while the shorter silent periods may reflect mainly defective inhibitory processes [32]. The result from paired pulse-TMS studies also supported the last hypothesis [22, 23, 35]; for example, Frasson et al. [22] showed that, in patients with CBD, paired magnetic stimuli delivered at short (inhibitory) interstimulus intervals (ISIs) invariably elicited enlarged MEPs; moreover, asymmetric corticocortical disinhibition $[22,36]$, as well as asymmetric TMS maps organization $[32,37]$, has been observed in patients with CBD.

In conclusion, several mechanisms could explain this abnormal motor cortical excitability, namely, loss of inhibitory neurons in the cortex or thalamus, effect of morphological changes in cortical neurons mainly in the somatosensory cortices, disruption of some neuronal circuits, or the existence of alternative cortical-subcortical pathways $[33,38]$. Further electrophysiological studies are necessary to better circumstantiate these hypothesis.

\subsection{Imaging in Corticobasal Degeneration. Morphologic} imaging of the brain, although normal in the early phases of the disease, may demonstrate asymmetrical cortical atrophy, in particular of the frontal and parietal lobe, more evident contralaterally to the side most severely clinically affected $[39,40]$. Asimmetrical atrophy in the basal ganglia, corpus callosum, lateral ventricles, and cerebral peduncles may be present.

Functional imaging studies might be useful in the differential diagnosis of patients with suspected CBD, showing asymmetrical hypoperfusion on SPECT and asymmetrical hypometabolism on PET involving the parietal-frontal cortex and basal ganglia [41].

Other neurodegenerative disorders sometimes overlap the $\mathrm{CBD}$, making its clinical diagnosis difficult. All criteria stress the combination of an akinetic-rigid syndrome with apraxia, alien limb syndrome, and cortical sensory deficits. A universally recognized feature is the asymmetry of clinical presentation, further corroborated by a contralateral asymmetrical atrophy on the structural and hypometabolism on the functional neuroimaging.

3.4. Neuropathology in Corticobasal Degeneration. Neuropathologically CBD presents as asymmetrical focal atrophy of the cerebral cortex focused on the peri-Rolandic posterior frontal and parietal cortex, especially the motor and sensory areas [42]. There is a relative sparing of temporal and occipital cortex, except in some forms presenting with dementia or primary progressive aphasia, which are characterised by a more symmetric and more severe involvement of the frontal and temporal lobes [6].

Basal ganglia are also involved with substantial atrophy in the lateral two-thirds of the substantia nigra, and, to a lesser extent, of putamen, pallidum, thalamus, and hypothalamus [43].

Histologically CBD is characterised by large pale ballooned neurons (neuronal achromasia), with tau-positive cytoplasmatic inclusions and astrocytic plaques (annular clusters of tau-positive deposits within the distal processes of astrocytes), typically distributed in atrophic cortices $[5,6$, 42].

Molecularly CBD is a taupathy, characterised by accumulation of abnormal filamentous inclusions of hyperphosphorylated tau-protein in neurons and glia, similarly to progressive supranuclear palsy (PSP), and some forms of frontotemporal dementia with parkinsonism (FTD) $[5,44]$. This molecular overlap, especially with PSP is very argued, and whether they are the extremities of the spectrum of a single disorder or two different disorders with a similar genetic predisposition is not clear $[3,18]$.

\subsection{Diagnostic Criteria and Growing Importance of Cog-} nitive Symptoms. The symptoms can be gathered in four categories: natural history and presentation, motor, sensory motor, and cognitive symptoms. The first three categories include characteristics which have been taken into account in almost all the previous diagnostic criteria. Up to 1994, dementia was an exclusion criterion of CBD; from 2003, on the base of new criteria, cognitive impairments support diagnosis, so the inclusion of the cognitive criteria reflects the growing recognition of the importance of cognitive assessment in the diagnosis of CBD $[3,45]$.

The core features of disease are insidious onset and progressive course, no identifiable cause (tumor, infarct) of symptomatology, cortical dysfunction includes at east one of the following: (i) focal or asymmetric ideomotor apraxia, (ii) alien-limb phenomena, (iii) cortical sensory loss, (iv) visual or sensory hemineglect, constructional apraxia, (v) focal 
or asymmetric myoclonus, (vi) apraxia of speech/nonfluent aphasia: extrapyramidal dysfunction as reflected by one of the following: (i) focal or asymmetrical appendicular rigidity lacking prominent and sustained L-dopa response, (ii) focal or asymmetrical appendicular dystonia.

The supportive investigations are variable degrees of focal or lateralized cognitive dysfunction, with relative preservation of learning and memory, on neuropsychometric testing, focal or asymmetric atrophy on computed tomography or magnetic resonance imaging, typically maximal in parietofrontal cortex, focal or asymmetric hypoperfusion on single-photon emission computed tomography and positron emission tomography, typically maximal in parietofrontal cortex/basal ganglia/thalamus [3].

3.6. Differential Diagnosis. Predominant parkinsonian features might not be easy to distinguish from idiopathic PD and atypical parkinsonian syndromes (e.g., PSP and multiple system atrophy, MSA). Akinetic-rigid syndrome, early inbalance and poor response to dopaminergic treatment are typical symptoms for CBP, PSP, and MSA, in contrast with PD. PSP is clinically and pathologically related to CBD, but with differences in symmetrical versus asymmetrical parkinsonism, and in the pattern of rigidity, which tends to be more nuchal/axial in PSP and more limb-accentuated in CBD. Also, while the superanuclear gaze palsy can occur in both conditions, in CBD it tends to affect the horizontal more than the vertical gaze and the saccade latency (delay in initiating saccades) more than saccade velocity (the speed of saccades).

In patients with cognitive presentation, the diagnosis depends on the specific case: pronounced visuoper-ceptual deficits related to dementia with Lewy bodies (DLB), language involvement to primary progressive aphasia (PPA), and prominent behavioral features to frontotemporal dementia (FTD) [3].

\section{Conclusion}

CBD can present with various clinical syndromes. The diagnosis of this heterogeneous disorder is difficult and misdiagnoses are frequent. It is important to explain the nature of the motor as well as the cognitive deficits to the patients as well as to all people involved in their care.

As a motor disorder, CBD has been recognized for over 30 years. However, more recent findings suggest that there is a neuropsychological syndrome associated with CBD. The most prominent neuropsychological features of CBD include limb apraxia, ideomotor and ideational apraxias. Executive function and language impairments are also reported.

The characterization of the natural history of patients with the CBD (clinical, laboratory, neuropsychological, radiological features) is important to improve the accuracy of diagnosis.

\section{References}

[1] J. Rebeiz, E. Kolodny, and E. Richardson, "Corticodentatonigral degeneration with neuronal achromasia: a progressive disorder of late adult life," Transactions of the American Neurological Association, vol. 92, pp. 23-26, 1967.

[2] P. Santacruz, L. Torner, F. Cruz-Sànchez, F. Lomena, A. Catafau, and R. Blesa, "Corticobasal degeneration syndrome: a case of Lewy body variant of Alzheimer's disease," International Journal of Geriatric Psychiatry, vol. 11, no. 6, pp. 559564, 1996.

[3] R. K. Mahapatra, M. J. Edwards, J. M. Schott, and K. P. Bhatia, "Corticobasal degeneration," Lancet Neurology, vol. 3, no. 12, pp. 736-743, 2004.

[4] B. F. Boeve, A. E. Lang, and I. Litvan, "Corticobasal degeneration and its relationship to progressive supranuclear palsy and frontotemporal dementia," Annals of Neurology, vol. 54, supplement 5, pp. S15-S19, 2003.

[5] M. Hasegawa, "Biochemistry and molecular biology of tauopathies," Neuropathology, vol. 26, no. 5, pp. 484-490, 2006.

[6] P. M. Wadia and A. E. Lang, "The many faces of corticobasal degeneration," Parkinsonism and Related Disorders, vol. 13, supplement 3, pp. S336-S340, 2007.

[7] G. K. Wenning, I. Litvan, J. Jankovic et al., "Natural history and survival of 14 patients with corticobasal degeneration confirmed at post-mortem examination," Journal of Neurology Neurosurgery and Psychiatry, vol. 64, no. 2, pp. 184-189, 1998.

[8] J. O. Rinne, M. S. Lee, P. D. Thompson, and C. D. Marsden, "Corticobasal degeneration: a clinical study of 36 cases," Brain, vol. 117, no. 5, pp. 1183-1196, 1994.

[9] J. A. Schneider, R. L. Watts, M. Gearing, R. P. Brewer, and S. S. Mirra, "Corticobasal degeneration: neuropathologic and clinical heterogeneity," Neurology, vol. 48, no. 4, pp. 959-969, 1997.

[10] K. Kompoliti, C. G. Goetz, B. F. Boeve et al., "Clinical presentation and pharmacological therapy in corticobasal degeneration," Archives of Neurology, vol. 55, no. 7, pp. 957-961, 1998.

[11] I. Litvan, D. A. Grimes, and A. E. Lang, "Phenotypes and prognosis: clinicopathologic studies of corticobasal degeneration," Advances in Neurology, vol. 82, pp. 183-196, 2000.

[12] Z. F. Vanek and J. Jankovic, "Dystonia in corticobasal degeneration," Advances in Neurology, vol. 82, pp. 61-67, 2000.

[13] N. Belfor, S. Amici, A. L. Boxer et al., "Clinical and neuropsychological features of corticobasal degeneration," Mechanisms of Ageing and Development, vol. 127, no. 2, pp. 203-207, 2006.

[14] S. Rivaud-Pechoux, M. Vidailhet, G. Gallouedec, I. Litvan, B. Gaymard, and C. Pierrot-Deseilligny, "Longitudinal ocular motor study in corticobasal degeneration and progressive supranuclear palsy," Neurology, vol. 54, no. 5, pp. 1029-1032, 2000.

[15] A. E. Lang, B. F. Boeve, and C. Bergeron, "Corticobasal degeneration," in Parkinson's Disease and Movement Disorders, J. J. Jankovic and E. Tolosa, Eds., pp. 186-202, Lippincott and Williams, 2006.

[16] K. Kompoliti, C. G. Goetz, B. F. Boeve et al., "Clinical presentation and pharmacological therapy in corticobasal degeneration," Archives of Neurology, vol. 55, no. 7, pp. 957961, 1998.

[17] N. L. Graham, T. H. Bak, and J. R. Hodges, "Corticobasal degeneration as a cognitive disorder," Movement Disorders, vol. 18, no. 11, pp. 1224-1232, 2003.

[18] A. E. Lang, "Corticobasal degeneration: selected developments," Movement Disorders, vol. 18, supplement 6, pp. S51S56, 2003. 
[19] J. L. Cummings and I. Litvan, "Neuropsychiatric aspects of corticobasal degeneration," Advances in Neurology, vol. 82, pp. 147-152, 2000.

[20] T. Mizuno, Y. Takanashi, T. Nakase et al., "Clinical application of magnetoencephalography in a patient with corticobasal degeneration," Journal of Neuroimaging, vol. 9, no. 1, pp. 4547, 1999.

[21] P. Grosse, A. Kuhn, C. Cordivari et al., "Coherence analysis in the myoclonus of corticobasal degeneration," Movement Disorders, vol. 18, no. 11, pp. 1345-1350, 2003.

[22] E. Frasson, L. Bertolasi, V. Bertasi et al., "Paired transcranial magnetic stimulation for the early diagnosis of corticobasal degeneration," Clinical Neurophysiology, vol. 114, no. 2, pp. 272-278, 2003.

[23] K. J. Huang, M. K. Lu, A. Kao, and C. H. Tsai, "Clinical, imaging and electrophysiological studies of corticobasal degeneration," Acta Neurologica Taiwanica, vol. 16, no. 1, pp. 13-21, 2007.

[24] C. Ozsancak, P. Auzou, and D. Hannequin, "La dégéné rescence corticobasale," Revue Neurologique, vol. 155, no. 12, pp. 1007-1020, 1999.

[25] J. Vion-Dury, N. Rochefort, P. Michotey, D. Planche, and M. Ceccaldi, "Proton magnetic resonance neurospectroscopy and EEG cartography in corticobasal degeneration: correlations with neuropsychological signs," Journal of Neurology, Neurosurgery and Psychiatry, vol. 75, no. 9, pp. 1352-1355, 2004.

[26] C. S. Lu, A. Ikeda, K. Terada et al., "Electrophysiological studies of early stage corticobasal degeneration," Movement Disorders, vol. 13, no. 1, pp. 140-146, 1998.

[27] P. D. Thompson, "Myoclomis in corticobasal degeneration," Clinical Neuroscience, vol. 3, pp. 203-208, 1996.

[28] D. Monza, C. Ciano, V. Scaioli et al., "Neurophysiological features in relation to clinical signs in clinically diagnosed corticobasal degeneration," Neurological Sciences, vol. 24, no. 1, pp. 16-23, 2003.

[29] E. R. Brunt, T. W. van Weerden, J. Pruim et al., "Unique myoclonic pattern in corticobasal degeneration," Movement Disorders, vol. 10, no. 2, pp. 132-142, 1995.

[30] R. Cantello, M. Gianelli, D. Bettucci et al., "Parkinson's disease rigidity: magnetic motor evoked potentials in a small hand muscle," Neurology, vol. 41, no. 9, pp. 1449-1456, 1991.

[31] W. Triggs, R. Macdoneii, D. Cros, K. Chiappa, B. Shahani, and B. Day, "Motor inhibition and excitation are independent effect of magnetic cortical stimulation," Annals of Neurology, vol. 32, no. 3, pp. 345-351, 1992.

[32] P. D. Thompson, B. L. Day, J. C. Rothwell, P. Brown, T. C. Britton, and C. D. Marsden, "The myoclonus in corticobasal degeneration: evidence for two forms of cortical reflex myoclonus," Brain, vol. 117, no. 5, pp. 1197-1207, 1994.

[33] A. Strafella, P. Ashby, and A. E. Lang, "Reflex myoclonus in cortical- basal ganglionic degeneration involves a transcortical pathway," Movement Disorders, vol. 12, no. 3, pp. 360-369, 1997.

[34] T. Yokota, Y. Saito, and Y. Shimizu, "Increased corticomotoneuronal excitability after peripheral nerve stimulation in dopa-nonresponsive hemiparkinsonism," Journal of the Neurological Sciences, vol. 129, no. 1, pp. 34-39, 1995.

[35] R. Hanajima, Y. Ugawa, Y. Terao, K. Ogata, and I. Kanazawa, "Ipsilateral cortico-cortical inhibition of the motor cortex in various neurological disorders," Journal of the Neurological Sciences, vol. 140, no. 1-2, pp. 109-116, 1996.

[36] Y. Okuma, T. Urabe, H. Mochizuki et al., "Asymmetric corticocortical inhibition in patients with progressive limb-kinetic apraxia," Acta Neurologica Scandinavica, vol. 102, no. 4, pp. 244-248, 2000.

[37] J. Valls-Solé, F. Tolosa, M. J. Marti et al., "Examination of motor output pathways in patients with corticobasal ganglionic degeneration using transcranial magnetic stimulation," Brain, vol. 124, no. 6, pp. 1131-1137, 2001.

[38] R. C. Leiguarda, M. Merello, M. I. Nouzeilles et al., "Limbkinetic apraxia in corticobasal degeneration: clinical and kinematic features," Movement Disorders, vol. 18, no. 1, pp. 49-59, 2003.

[39] M. Savoiardo, M. Grisoli, and F. Girotti, "Magnetic resonance imaging in CBD, related atypical parkinsonian disorders, and dementias," Advances in Neurology, vol. 82, pp. 197-208, 2000.

[40] P. Soliveri, D. Monza, D. Paridi et al., "Cognitive and magnetic resonance imaging aspects of corticobasal degeneration and progressive supranuclear palsy," Neurology, vol. 53, no. 3, pp. 502-507, 1999.

[41] D. J. Brooks, "Functional imaging studies in corticobasal degeneration," Advances in Neurology, vol. 82, pp. 209-215, 2000.

[42] D. W. Dickson, C. Bergeron, S. S. Chin et al., "Office of rare diseases neuropathologic criteria for corticobasal degeneration," Journal of Neuropathology and Experimental Neurology, vol. 61, no. 11, pp. 935-946, 2002.

[43] G. Kłodowska-Duda, J. Słowinski, G. Opala et al., "Corticobasal degeneration-clinico-pathological considerations," Folia Neuropathologica, vol. 44, no. 4, pp. 257-264, 2006.

[44] H. R. Morris, A. J. Lees, and N. W. Wood, "Neurofibrillary tangle Parkinsonian disorders-tau pathology and tau genetics," Movement Disorders, vol. 14, no. 5, pp. 731-736, 1999.

[45] H. T. Bak, "Corticobasal degeneration: clinical aspects," in Handbook of Clinical Neurology, vol. 89 (3rd series) Dementias, pp. 509-521, 2008. 


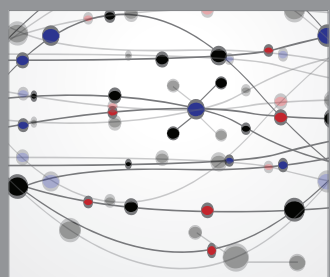

The Scientific World Journal
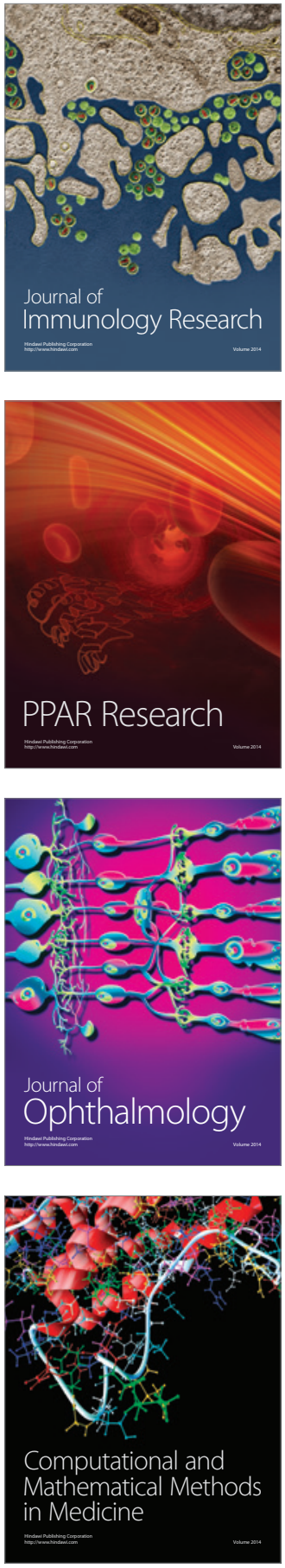

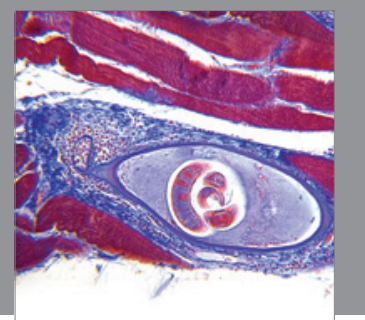

Gastroenterology

Research and Practice
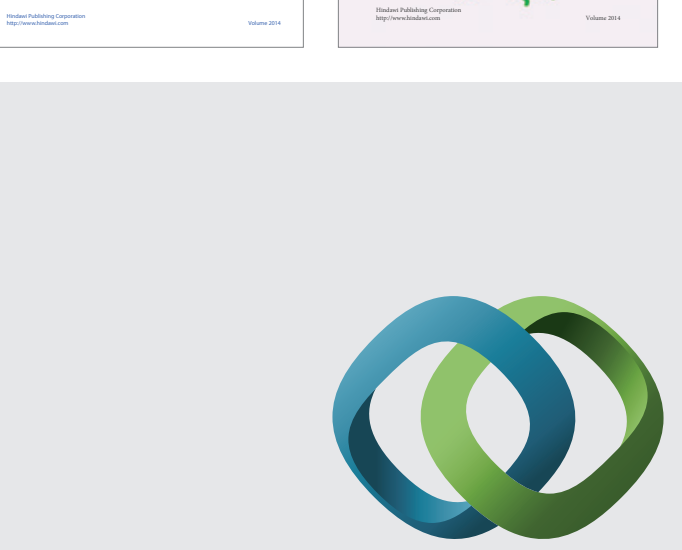

\section{Hindawi}

Submit your manuscripts at

http://www.hindawi.com
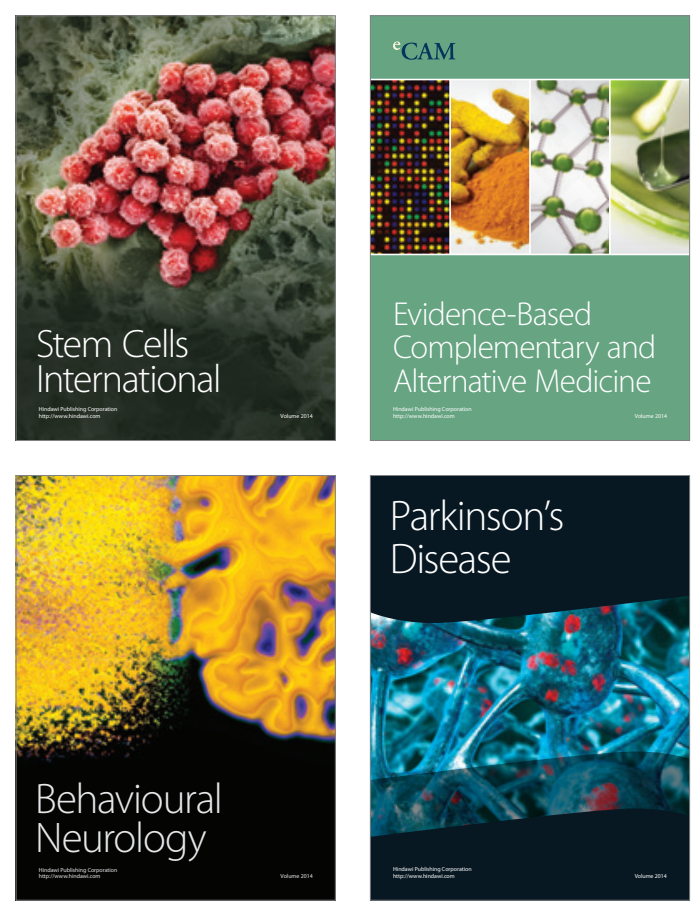

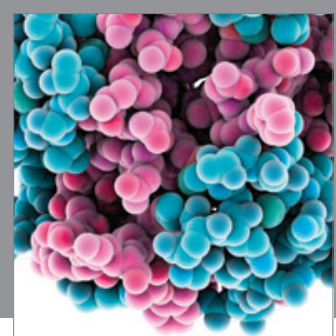

Journal of
Diabetes Research

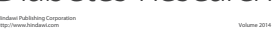

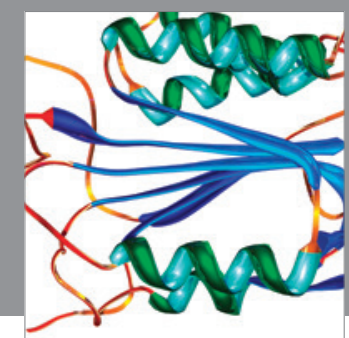

Disease Markers
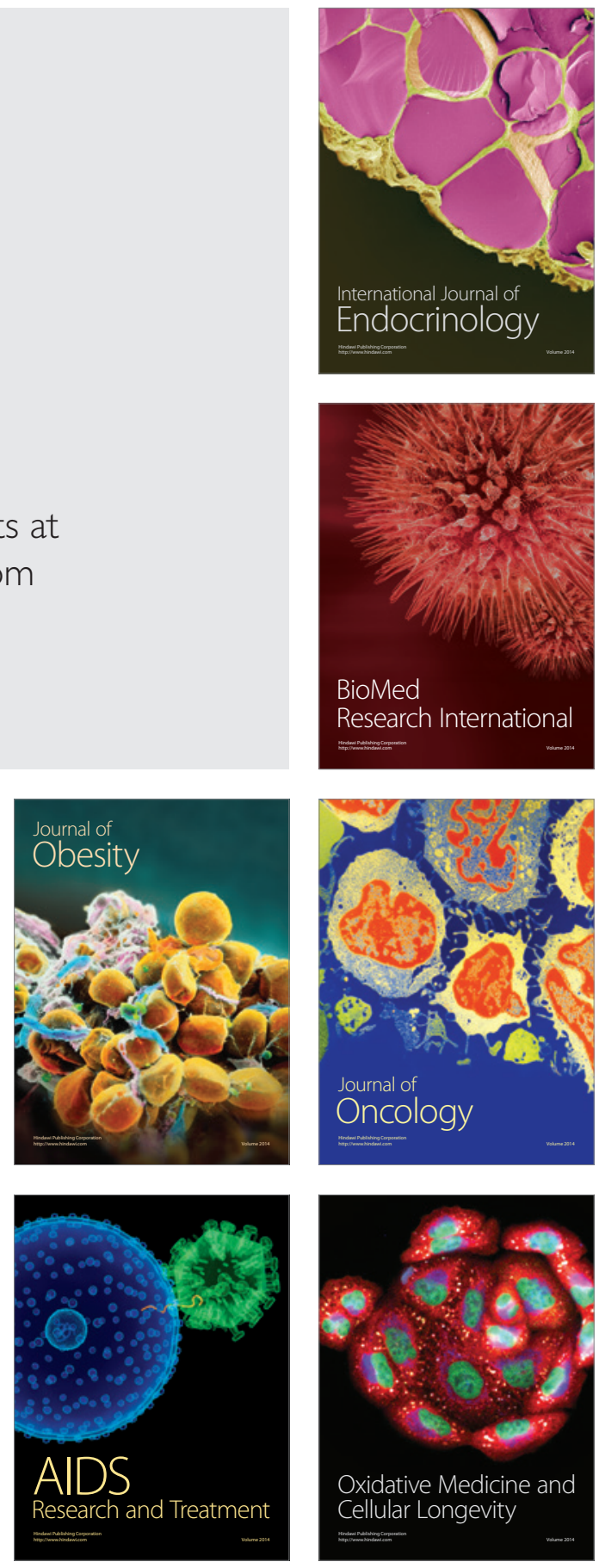